\title{
Effect of dietary conjugated linoleic acid (CLA) on the growth and lipid metabolism of geese and fatty acid composition of their tissues
}

\author{
Xu-hui Zhang1, Bao-wei Wang ${ }^{1 \#}$, Lei Wang1, Fang-yu Long1, Zhi-gang Yang1, \\ Shi-hao Yu ${ }^{1}$, Ya-chao Wang ${ }^{1}$, Xiao-xiao Wei ${ }^{1}$, Li-zhen Jing ${ }^{1}$ and Guang-lei Liu ${ }^{2}$ \\ ${ }^{1}$ Waterfowl Research Institute, Qingdao Agricultural University, Shandong Qingdao 266109, P.R. China \\ ${ }^{2}$ Institute of Animal Husbandry, Chinese Academy of Agricultural Sciences, Beijing, 100094, P.R. China
}

\begin{abstract}
The objective of this study was to determine the effects of conjugated linoleic acid (CLA) oil supplementation on the growth performance and lipid metabolism in geese and the fatty acid concentrations in their liver and muscle tissues. One hundred and ninety two one-day old geese were randomly assigned to one of four dietary treatments. The diet in Group A, the control, contained $2.5 \%$ soyabean oil, that in Group B 2.0\% soyabean oil and 0.5\% CLA, that in Group C 1.0\% soybean oil and 1.5\% CLA and that in Group D 2.5\% CLA. The birds were fed for 56 days. No significant effects were observed in body weight and body weight gain between Groups B, C and the control, but these parameters were significantly lower in Group D compared to the control. The feed intake, feed conversion ratio (FCR) and abdominal fat percentage (AFP) were significantly lower in the groups receiving CLA in their diets compared with the control. Dietary CLA altered serum lipid concentrations by decreasing total cholesterol, triglyceride and low density lipoprotein-cholesterol concentrations, the atherogenic index and activity of lipoprotein lipase, and increased serum concentration of high density lipoprotein-cholesterol. The fatty acid composition of the liver and muscle tissues showed significant increases in the biologically active cis- 9 , trans- 11 and trans- 10 , cis- 12 CLA isomers in the geese fed increasing levels of CLA. The supplementation of CLA to the geese led to significant increases in saturated fatty acid concentrations and significant reductions in the monounsaturated fatty acid concentrations in liver and muscle tissues. The results clearly demonstrated that geese can successfully incorporate CLA in their liver and muscle tissues, thus producing a healthy food for humans.
\end{abstract}

Keywords: Conjugated linoleic acid, geese, growth, lipid metabolism, fatty acid composition

${ }^{\#}$ Corresponding author. E-mail: wangbw@qau.edu.cn

\section{Introduction}

Conjugated linoleic acid (CLA) is a term used to describe positional and geometric isomers of linoleic acid (18:2n-6; LA). The two main naturally occurring isomers are cis-9, trans-11 and trans-10, cis- 12 . These compounds occur particularly in dairy products and ruminant meat, such as beef and lamb, but are present at lower concentrations in many foodstuffs (Chin et al., 1992; Pariza et al., 2001).

Conjugated linoleic acid has received much attention over the past decade because of its multiple biological effects. Among them, these fatty acids have been shown to reduce body fat accumulation (Badinga et al., 2003; Yamasaki et al., 2003; Wang \& Jones, 2004), to improve feed efficiency (Li \& Watkins, 1998) in several rodent models and to lower blood lipids and atherogenic risk in animal studies. Indeed, early work demonstrated that CLA reduces adiposity in growing mice, where 6-week old ICR mice were fed a diet containing less than 1\% CLA for 28-32 days (Park et al., 1997). Further work in various animal models confirmed those findings (Delany et al., 1999). Feeding non-obese mice with CLA reduced fat mass in some depots, specifically retroperitoneal and epididymal white adipose tissue masses and brown adipose tissue (Tsuboyama-Kasaoka et al., 2000), suggesting that they are more sensitive to CLA-mediated effects. In addition, when feeding rabbits with atherogenic diets the CLA mixtures resulted in less early aortic atherosclerosis (Lee et al., 1994) or in regression of preexisting atherosclerosis (Kritchevsky et al., 2000). Feeding different levels of CLA to hamsters led to lower total plasma cholesterol, low density lipoprotein cholesterol (LDL-C) and triglyceride concentrations (Nicolosi et al., 1997). Proposed anti-obesity mechanisms of CLA include decreased energy/food intake and increased energy expenditure (Ohnuki et al., 2001; Terpstra et al., 2002), decreased pre-adipocyte differentiation and proliferation (Evans et al., 2002), decreased lipogenesis (Brown et al., 2001; Oku et al., 2003) and increased lipolysis and fatty acid oxidation 
(Takahashi et al., 2003). The effects of dietary CLA in the growth performance and flesh quality of waterfowl have not been reported. Therefore, a further increase in the dietary CLA content could be of interest in enhancing the nutritional value and decreasing the fat deposition of geese destined for human consumption.

In light of the above, the aim of the present study was to investigate the effects of graded dietary levels of CLA $(0 \%, 0.5 \%, 1.5 \%$ and $2.5 \%)$ on growth performance, lipid metabolism and fatty acid composition in the liver, leg and breast tissues of geese. The trial was performed with the hypothesis that the supplementation of geese diets with CLA would produce more healthy geese products for human consumption.

\section{Materials and Methods}

The experiment was carried out in an experimental animal house at the Qingdao Agricultural University, Shandong, China and lasted 56 days. A total of 192 one-day old Wulong geese with a mean initial body weight of $79 \pm 1.13 \mathrm{~g}$ ( \pm s.d.) was selected and randomly assigned to one of four dietary treatments. Each treatment consisted of four replicates of 12 geese. The geese were fed a maize-soyabean basal diet containing $2.5 \%$ oil (Table 1 ). The different treatments contained different proportions of soya oil and CLA.

Table 1 Ingredients and calculated composition of the experimental diets containing different levels of conjugated linoleic acid (CLA) (as fed basis)

\begin{tabular}{|c|c|c|c|c|c|c|c|c|}
\hline & \multicolumn{4}{|c|}{ 0-28 days } & \multicolumn{4}{|c|}{ 29-56 days } \\
\hline & \multicolumn{8}{|c|}{ Dietary treatments $^{2}$} \\
\hline & A & B & $\mathrm{C}$ & $\mathrm{D}$ & $\mathrm{A}$ & $\mathrm{B}$ & $\mathrm{C}$ & $\mathrm{D}$ \\
\hline \multicolumn{9}{|l|}{ Formulation (\%) } \\
\hline Maize & 59.0 & 59.0 & 59.0 & 59.0 & 54.9 & 54.9 & 54.9 & 54.9 \\
\hline Soyabean meal & 30.5 & 30.5 & 30.5 & 30.5 & 24.0 & 24.0 & 24.0 & 24.0 \\
\hline Chinese wildrye meal & 4.5 & 4.5 & 4.5 & 4.5 & 15.0 & 15.0 & 15.0 & 15.0 \\
\hline Soyabean oil & 2.5 & 2.0 & 1.0 & 0 & 2.5 & 2.0 & 1.0 & 0 \\
\hline CLA oil & 0 & 0.5 & 1.5 & 2.5 & 0 & 0.5 & 1.5 & 2.5 \\
\hline Limestone & 0.9 & 0.9 & 0.9 & 0.9 & 0.9 & 0.9 & 0.9 & 0.9 \\
\hline $\mathrm{CaHPO}_{4}$ & 1.32 & 1.32 & 1.32 & 1.32 & 1.40 & 1.40 & 1.40 & 1.40 \\
\hline $\mathrm{NaCl}$ & 0.30 & 0.30 & 0.30 & 0.30 & 0.35 & 0.35 & 0.35 & 0.35 \\
\hline Premix $^{1}$ & 1.0 & 1.0 & 1.0 & 1.0 & 1.0 & 1.0 & 1.0 & 1.0 \\
\hline DL-Methionine (g/kg) & 1.4 & 1.4 & 1.4 & 1.4 & 1.4 & 1.4 & 1.4 & 1.4 \\
\hline Lysine $(\mathrm{g} / \mathrm{kg})$ & 0 & 0 & 0 & 0 & 1.5 & 1.5 & 1.5 & 1.5 \\
\hline \multicolumn{9}{|l|}{ Calculated composition } \\
\hline Dry matter, $\mathrm{g} / \mathrm{kg}$ & 900 & 900 & 900 & 900 & 900 & 900 & 900 & 900 \\
\hline Metabolisable energy, $\mathrm{MJ} / \mathrm{kg}$ & 11.7 & 11.7 & 11.7 & 11.7 & 10.9 & 10.9 & 10.9 & 10.9 \\
\hline Crude protein $(\mathrm{mg} / \mathrm{kg})$ & 189.3 & 189.3 & 189.3 & 189.3 & 164.1 & 164.1 & 164.1 & 164.1 \\
\hline $\mathrm{NaCl}(\mathrm{mg} / \mathrm{kg})$ & 3.0 & 3.0 & 3.0 & 3.0 & 3.5 & 3.5 & 3.5 & 3.5 \\
\hline Crude fibre (mg/kg) & 42 & 42 & 42 & 42 & 65 & 65 & 65 & 65 \\
\hline Calcium (mg/kg) & 8 & 8 & 8 & 8 & 8 & 8 & 8 & 8 \\
\hline Available phosphorus (g/kg) & 0.4 & 0.4 & 0.4 & 0.4 & 0.4 & 0.4 & 0.4 & 0.4 \\
\hline Lysine $(\mathrm{g} / \mathrm{kg})$ & 0.95 & 0.95 & 0.95 & 0.95 & 0.91 & 0.91 & 0.91 & 0.91 \\
\hline Methionine+Cystine (g/kg) & 0.74 & 0.74 & 0.74 & 0.74 & 0.69 & 0.69 & 0.69 & 0.69 \\
\hline
\end{tabular}

${ }^{1}$ Containing per kilogram of diet: $1500 \mathrm{IU}$ vitamin $\mathrm{A} ; 200 \mathrm{IU}$ cholecalciferol; $12.5 \mathrm{mg}$ vitamin $\mathrm{E} ; 1.5 \mathrm{mg}$ menadione; $2.2 \mathrm{mg}$ thiamine; $5.0 \mathrm{mg}$ riboflavin; $65 \mathrm{mg}$ niacin; $15 \mathrm{mg}$ pantothenic acid; $2 \mathrm{mg}$ pyridoxine; $0.2 \mathrm{mg}$ biotin, $0.5 \mathrm{mg}$ folic acid; $500 \mathrm{mg}$ alkali; $96 \mathrm{mg} \mathrm{Fe} ; 5 \mathrm{mg} \mathrm{Cu} ; 66 \mathrm{mg} \mathrm{Mn} ; 60 \mathrm{mg} \mathrm{Zn;} 0.42 \mathrm{mg} \mathrm{I} ; 0.15 \mathrm{mg}$ Se.

${ }^{2}$ Dietary treatments: Group A - dietary CLA at an inclusion level of 0\%; Group B - dietary CLA at an inclusion level of $0.5 \%$; Group C - dietary CLA at an inclusion level of $1.5 \%$; Group D - dietary CLA at an inclusion level of $2.5 \%$.

The control diet (Group A) contained 2.5\% soya oil and 0\% CLA, and in the other treatments CLA replaced the soya oil. In Group B the diet contained 0.5\% CLA, that in Group C 1.5\% CLA and in Group D 
2.5\% CLA and no soya oil. The CLA contained 80\% CLA free fatty acids mainly consisting of $36.7 \%$ cis 9 , trans 11 and $39.5 \%$ trans 10, cis 12 isomers, and was purchased from Qingdao Aohai Biologic Limited Company, Shandong, China. The fatty acid profiles of the experimental diets are presented in Table 2.

The geese were fed a starter diet until $28 \mathrm{~d}$ of age followed by a grower diet from 29 to $56 \mathrm{~d}$. The diets were formulated according to the nutritional requirements for geese (P.R. the Act of Technical Specification for Wulong-geese Production; Serial Number: DB37/T503-2004, 2004) and were formulated to be isoenergetic, isolipidic and isonitrogeneous (Table 1). Ingredients were obtained from a local market in Qingdao Shandong, China. An antioxidant, butylated hydroxytoluene (BHT) was added to minimize oxidation of the fatty acids. Diet preparations were carried out at the experimental animal house.

Standard management practices for growing geese were followed. The birds had free access to their feed and water, and immunoprophylaxis was applied. All experimental procedures were performed according to the Guide for Animal Care and Use of Laboratory Animals of the Institutional Animal Care and Use Committee of the university and the protocol was approved by the Animal Ethics Committee.

Table 2 Main fatty acids and conjugated linoleic acid (CLA) isomers ( $\%$ total fatty acids) and total CLA (\% total lipids) of diets with different CLA incorporation levels $(0,0.5,1.5$ or $2.5 \%)$

\begin{tabular}{|c|c|c|c|c|c|c|c|c|}
\hline \multirow{3}{*}{$\begin{array}{l}\text { Fatty acid profile* } \\
(\%)\end{array}$} & \multicolumn{4}{|c|}{$0-28$ days } & \multicolumn{4}{|c|}{ 29-56 days } \\
\hline & \multicolumn{8}{|c|}{ Dietary treatments } \\
\hline & $\mathrm{A}$ & $\mathrm{B}$ & $\mathrm{C}$ & $\mathrm{D}$ & $\mathrm{A}$ & $\mathrm{B}$ & $\mathrm{C}$ & $\mathrm{D}$ \\
\hline C16:0 & 13.0 & 11.4 & 9.4 & 10.1 & 12.6 & 11.8 & 11.0 & 9.2 \\
\hline C18:0 & 5.2 & 4.4 & 3.4 & 2.4 & 5.1 & 4.4 & 3.6 & 3.8 \\
\hline C18:1, n-9 & 19.6 & 18.0 & 16.8 & 15.7 & 20.5 & 18.2 & 16.2 & 13.4 \\
\hline C18:2, n-6 & 53.8 & 51.6 & 42.6 & 34.6 & 53.4 & 51.2 & 43.2 & 35.4 \\
\hline$\alpha \mathrm{C} 18: 3, \mathrm{n}-3$ & 8.4 & 7.2 & 6.4 & 5.1 & 8.4 & 7.2 & 6.0 & 4.4 \\
\hline Total CLA & ND & 7.4 & 21.4 & 32.1 & ND & 7.2 & 20.2 & 33.8 \\
\hline c-9, t-11 CLA & ND & 3.4 & 10.3 & 15.3 & ND & 3.4 & 9.2 & 16.2 \\
\hline $\mathrm{t}-10, \mathrm{c}-12$ CLA & ND & 4.0 & 11.1 & 16.8 & ND & 3.8 & 11.0 & 17.6 \\
\hline
\end{tabular}

* All values are means as weight percentages of total fatty acid methyl esters.

ND - not detectable.

Data on feed intake and body weights of the geese were collected weekly and mortalities were recorded daily. At the end of the feeding trial feed was withheld for $12 \mathrm{~h}$ whereafter three geese were randomly selected from each replicate, weighed, and blood samples were collected into heparinized syringes from each bird via their wing veins. Serum was obtained within $3 \mathrm{~h}$ of sampling after centrifugation at $1000 \mathrm{x} \mathrm{g}$ for $10 \mathrm{~min}$ and at $4{ }^{\circ} \mathrm{C}$. Serum samples were frozen and stored at $-80{ }^{\circ} \mathrm{C}$ pending analyses. After the collection of blood, all geese were killed by exsanguination and slaughtered immediately. The liver, leg and breast muscle samples were removed, frozen immediately in liquid nitrogen and stored in a freezer at $-80{ }^{\circ} \mathrm{C}$ prior to fatty acid determinations. Thereafter abdominal fat was collected and weighed.

For determination of serum total cholesterol (TC), LDL-C, high density lipoprotein-cholesterol (HDL-C), total triglyceride (TG) concentrations and lipoprotein lipase (LPL) activities the corresponding diagnostic kits (Nanjing Jiancheng Bioengineering Institute, Nanjing, P.R. China) were used according to the instructions of the manufacturer. The lipoproteins, LDL-C and HDL-C, were fractionated by a dual precipitation technique (Wilson \& Spiger, 1973). After fractional precipitation, lipoprotein cholesterol was estimated. The atherogenic index (AI) was calculated as (TC-HDL-C)/HDL-C (Yang et al., 2006). A decreased value of AI showed a stronger lipid-lowering effect, as well as a high antiatherogenic potential.

Total lipids were extracted according to the method of Folch et al. (1957) and lipid content determined gravimetrically as described previously (Kennedy et al., 2005). Fatty acid methyl esters (FAME) from diets and tissue total lipids were prepared by the acid-catalyzed transesterification of total lipid, following the method of Christie (1982), except that the reaction was performed at $80{ }^{\circ} \mathrm{C}$ for $3 \mathrm{~h}$. Fatty acid methyl esters were extracted and purified by reaction with $4 \% \mathrm{HCl}$ in methanol for $20 \mathrm{~min}$ at $60{ }^{\circ} \mathrm{C}$. The FAME were 
separated and quantified by gas-liquid chromatography (Carlo Erba Vega 8160, Milan, Italy) using a $30 \mathrm{~m} \mathrm{x}$ $0.32 \mathrm{~mm}$ i.d. capillary column (CP Wax 52CB, Chrompak, London, U.K.) and on-column injection. Hydrogen was used as carrier gas and temperature programming was from $50{ }^{\circ} \mathrm{C}$ to $150{ }^{\circ} \mathrm{C}$ at $40{ }^{\circ} \mathrm{C} / \mathrm{min}$ and then to $230^{\circ} \mathrm{C}$ at $2.0^{\circ} \mathrm{C} / \mathrm{min}$. Methyl esters were identified and quantified as described previously (Kennedy et al., 2005). Individual fatty acid methyl esters were expressed as percentages of all peaks.

Unless otherwise stated, all data are presented as means \pm s.d. The effects of dietary treatments were determined by Student's t-test or one-way analysis of variance (ANOVA) followed, where appropriate, by the Tukey's comparison test (SAS, 2001). Percentage data and data which were identified as nonhomogeneous (Bartlett's test) were subjected to arcsine transformation before analysis. Significance was evaluated at the 0.05 level.

\section{Results}

The effect of dietary supplementation of CLA on body weight, weight gain, feed intake and feed conversion ratio (FCR) of the birds is presented in Table 3. Differences with respect to body weight and weight gain during the two growth phases of $0-28$ and $29-56 \mathrm{~d}$ were significant $(\mathrm{P}<0.05)$ between Groups $\mathrm{A}$ and $\mathrm{D}$, but not $(\mathrm{P}>0.05)$ between Groups A (the control), B and C. Group D exhibited the best FCR compared to the other groups $(\mathrm{P}<0.01)$, and the lowest $(\mathrm{P}<0.01)$ AFP compared with the control. Feed intake was lower $(\mathrm{P}<0.01)$ in Group $\mathrm{D}$ than in the other groups.

Table 3 The effects of dietary conjugated linoleic acid (CLA) treatments on growth performance of geese

\begin{tabular}{lrrrr}
\hline & \multicolumn{4}{c}{ Dietary treatments } \\
\cline { 2 - 5 } & Group A & Group B & Group C & Group D \\
& CLA (\%) & \multicolumn{1}{c}{0} & 0.5 & 1.5 \\
\hline 0-28 days & $1143^{\mathrm{a}} \pm 65.6$ & $1204^{\mathrm{a}} \pm 74.6$ & $1201^{\mathrm{a}} \pm 71.6$ & $795^{\mathrm{b}} \pm 57.3$ \\
Body weight, g & $1064^{\mathrm{a}} \pm 66.4$ & $1126^{\mathrm{a}} \pm 74.2$ & $1122^{\mathrm{a}} \pm 72.0$ & $715^{\mathrm{b}} \pm 56.3$ \\
Weight gain, g & $2580^{\mathrm{a}} \pm 161.6$ & $2369^{\mathrm{b}} \pm 156.7$ & $2374^{\mathrm{b}} \pm 152.4$ & $1485^{\mathrm{c}} \pm 117.2$ \\
Feed intake, g & $2.43^{\mathrm{a}} \pm 0.01$ & $2.10^{\mathrm{b}} \pm 0.02$ & $2.12^{\mathrm{b}} \pm 0.02$ & $2.08^{\mathrm{b}} \pm 0.01$ \\
FCR & $0.53^{\mathrm{a}} \pm 0.05$ & $0.48^{\mathrm{a}} \pm 0.06$ & $0.37^{\mathrm{b}} \pm 0.05$ & $0.09^{\mathrm{c}} \pm 0.07$ \\
AFP (\%) & & & & \\
29-56 days & & & & \\
Body weight, g & $2719^{\mathrm{a}} \pm 156.2$ & $2947^{\mathrm{a}} \pm 189.4$ & $2913^{\mathrm{a}} \pm 173.9$ & $1763^{\mathrm{b}} \pm 142.2$ \\
Weight gain, g & $1624^{\mathrm{a}} \pm 138.7$ & $1787^{\mathrm{a}} \pm 169.2$ & $1792^{\mathrm{a}} \pm 152.1$ & $968^{\mathrm{b}} \pm 120.9$ \\
Feed intake, g & $6824^{\mathrm{a}} \pm 202.5$ & $5806^{\mathrm{b}} \pm 226.0$ & $5697^{\mathrm{b}} \pm 272.3$ & $2941^{\mathrm{c}} \pm 207.0$ \\
FCR & $4.20^{\mathrm{a}} \pm 0.01$ & $3.28^{\mathrm{b}} \pm 0.02$ & $3.29^{\mathrm{b}} \pm 0.01$ & $3.04^{\mathrm{b}} \pm 0.01$ \\
AFP (\%) & $1.57^{\mathrm{a}} \pm 0.13$ & $0.94^{\mathrm{b}} \pm 0.15$ & $0.84^{\mathrm{b}} \pm 0.09$ & $0.50^{\mathrm{c}} \pm 0.06$
\end{tabular}

$\overline{\mathrm{a}-\mathrm{c}}$ Means within rows with superscripts $\mathrm{a}-\mathrm{b}$ and $\mathrm{b}-\mathrm{c}$ signify differences at $\mathrm{P}<0.05$ and a-c differences at $\mathrm{P}<0.01$; FCR - feed conversion ratio ( $\mathrm{kg}$ feed intake/kg weight gain);

AFP - percentage of abdominal fat.

Table 4 presents the effect of dietary CLA treatments on serum lipid biochemical variables in the geese. Treatments with graded levels of CLA supplementation brought about a decrease $(\mathrm{P}<0.05)$ in the TG levels and AI of the geese, and an increase $(\mathrm{P}<0.05)$ in the HDL-C levels in comparison with the control. The diets containing dietary CLA at inclusion levels of $0.5 \%$ and $1.5 \%$ reduced $(\mathrm{P}<0.05)$ the TC levels in serum, and at $1.5 \%$ and $2.5 \%$ CLA decreased $(\mathrm{P}<0.05)$ the serum LDL-C concentration in geese compared with birds in the unsupplemented control. Addition of different levels of CLA to the diet increased the activity of LPL. 
Table 4 The effects of dietary conjugated linoleic acid (CLA) treatments on serum lipid biochemical variables in geese

\begin{tabular}{ccccccc}
\hline Dietary treatments & $\begin{array}{c}\text { TG } \\
(\mathrm{mmol} / \mathrm{L})\end{array}$ & $\begin{array}{c}\text { TC } \\
(\mathrm{mmol} / \mathrm{L})\end{array}$ & $\begin{array}{c}\text { LDL-C } \\
(\mathrm{mmol} / \mathrm{L})\end{array}$ & $\begin{array}{c}\text { HDL-C } \\
(\mathrm{mmol} / \mathrm{L})\end{array}$ & AI & $\begin{array}{c}\text { LPL } \\
(\mathrm{U} / \mathrm{mL})\end{array}$ \\
\hline Group A & $0.75^{\mathrm{a}} \pm 0.15$ & $5.13^{\mathrm{a}} \pm 0.39$ & $1.97^{\mathrm{a}} \pm 0.09$ & $2.39^{\mathrm{b}} \pm 0.57$ & $1.15^{\mathrm{a}} \pm 0.15$ & $1.18^{\mathrm{b}} \pm 0.09$ \\
Group B & $0.55^{\mathrm{b}} \pm 0.12$ & $4.53^{\mathrm{b}} \pm 0.54$ & $1.80^{\mathrm{ab}} \pm 0.10$ & $2.84^{\mathrm{a}} \pm 0.23$ & $0.60^{\mathrm{b}} \pm 0.12$ & $1.86^{\mathrm{a}} \pm 0.13$ \\
Group C & $0.53^{\mathrm{b}} \pm 0.09$ & $4.56^{\mathrm{b}} \pm 0.72$ & $1.62^{\mathrm{b}} \pm 0.11$ & $3.00^{\mathrm{a}} \pm 0.35$ & $0.52^{\mathrm{b}} \pm 0.09$ & $2.01^{\mathrm{a}} \pm 0.14$ \\
Group D & $0.56^{\mathrm{b}} \pm 0.13$ & $4.84^{\mathrm{ab}} \pm 0.41$ & $1.64^{\mathrm{b}} \pm 0.17$ & $2.95^{\mathrm{a}} \pm 0.30$ & $0.64^{\mathrm{b}} \pm 0.13$ & $1.90^{\mathrm{a}} \pm 0.11$
\end{tabular}

${ }^{\mathrm{a}-\mathrm{c}}$ Means within columns with superscripts a-b signify differences at $\mathrm{P}<0.05 ; \mathrm{n}=12$.

Group A - 0\% CLA; Group B - 0.5\% CLA; Group C - 1.5\% CLA Group D - 2.5\% CLA;

TG - total triglyceride; TC - total cholesterol; LDL-C - low density lipoprotein-cholesterol; HDL-C - high density lipoprotein - cholesterol; AI - atherogenic index; LPL - Lipoprotein lipase.

Table 5 The effects of dietary conjugated linoleic acid (CLA) treatments on the fatty acid composition of liver in geese

\begin{tabular}{|c|c|c|c|c|c|}
\hline \multirow{3}{*}{ Fatty acid } & \multirow[b]{3}{*}{ CLA $(\%)$} & \multicolumn{4}{|c|}{ Fatty acids (\% of total) } \\
\hline & & Group A & Group B & Group $\mathrm{C}$ & Group D \\
\hline & & 0 & 0.5 & 1.5 & 2.5 \\
\hline $\mathrm{C} 14: 0$ & & $2.40^{b} \pm 0.53$ & $2.42^{b} \pm 0.23$ & $3.32^{\mathrm{a}} \pm 0.20$ & $3.89^{\mathrm{a}} \pm 0.22$ \\
\hline C16:0 & & $22.82^{b} \pm 1.54$ & $23.73^{\mathrm{ab}} \pm 1.68$ & $25.38^{\mathrm{a}} \pm 2.09$ & $25.18^{\mathrm{a}} \pm 1.91$ \\
\hline $\mathrm{C} 16: 1, \mathrm{n}-7$ & & $3.14 \pm 0.65$ & $4.18 \pm 0.79$ & $3.40 \pm 0.46$ & $3.92 \pm 0.56$ \\
\hline C18:0 & & $5.64^{\mathrm{c}} \pm 0.56$ & $6.82^{b} \pm 0.75$ & $7.23^{b} \pm 0.69$ & $9.38^{\mathrm{a}} \pm 0.76$ \\
\hline C18:1, n-9 & & $42.40^{\mathrm{a}} \pm 3.21$ & $41.90^{\mathrm{a}} \pm 2.54$ & $38.18^{b} \pm 2.95$ & $35.52^{b} \pm 2.84$ \\
\hline $\mathrm{C} 18: 2, \mathrm{n}-6$ & & $17.62^{\mathrm{a}} \pm 2.92$ & $15.38^{\mathrm{b}} \pm 3.29$ & $15.91^{b} \pm 2.73$ & $13.63^{c} \pm 1.56$ \\
\hline $\mathrm{c}-9, \mathrm{t}-11$ CLA & & ND & $0.37^{c} \pm 0.05$ & $0.81^{b} \pm 0.04$ & $1.57^{\mathrm{a}} \pm 0.07$ \\
\hline $\mathrm{t}-10, \mathrm{c}-12 \mathrm{CLA}$ & & ND & $0.32^{\mathrm{c}} \pm 0.02$ & $0.65^{b} \pm 0.03$ & $1.24^{\mathrm{a}} \pm 0.09$ \\
\hline$\alpha \mathrm{C} 18: 3, \mathrm{n}-3$ & & $1.02^{\mathrm{a}} \pm 0.13$ & $0.63^{b} \pm 0.15$ & $0.61^{\mathrm{b}} \pm 0.14$ & $0.50^{\mathrm{b}} \pm 0.10$ \\
\hline $\mathrm{C} 20: 4, \mathrm{n}-6$ & & $0.53 \pm 0.12$ & $0.45 \pm 0.07$ & $0.54 \pm 0.09$ & $0.49 \pm 0.04$ \\
\hline$\Sigma$ CLA & & ND & $0.69^{c} \pm 0.05$ & $1.46^{\mathrm{b}} \pm 0.42$ & $2.82^{\mathrm{a}} \pm 0.51$ \\
\hline$\Sigma$ SFA & & $30.86^{\mathrm{c}} \pm 3.49$ & $32.97^{\mathrm{c}} \pm 2.35$ & $35.93^{b} \pm 3.28$ & $38.45^{\mathrm{a}} \pm 3.78$ \\
\hline$\Sigma$ MUFA & & $45.54^{\mathrm{a}} \pm 4.29$ & $46.08^{a} \pm 3.97$ & $41.58^{b} \pm 4.02$ & $39.44^{c} \pm 4.18$ \\
\hline$\Sigma$ PUFA & & $19.17 \pm 1.24$ & $17.15 \pm 1.65$ & $18.52 \pm 1.89$ & $17.43 \pm 1.94$ \\
\hline UP & & $4.43 \pm 0.75$ & $3.80 \pm 0.77$ & $3.97 \pm 0.71$ & $4.68 \pm 0.44$ \\
\hline
\end{tabular}

\footnotetext{
${ }^{\mathrm{a}-\mathrm{c}}$ Means within rows with superscripts $\mathrm{a}-\mathrm{b}$ and $\mathrm{b}$-c signify differences at $\mathrm{P}<0.05$ and a-c differences at $\mathrm{P}<0.01$. $\mathrm{n}=12$.

$\Sigma$ SFA - total saturated fatty acids; $\Sigma$ MUFA - total monounsaturated fatty acids; $\Sigma$ PUFA - total polyunsaturated fatty acids; $\Sigma$ CLA - total conjugated linoleic acids; UP - unidentified peaks; ND - not detectable.
}

The fatty acid compositions of liver, leg and breast tissues are shown in Tables 5, 6 and 7, respectively. Supplementation of dietary CLA resulted in their deposition into lipids of the liver and muscle tissue, and an increase $(\mathrm{P}<0.01)$ in the biologically active cis-9, trans-11 and trans-10, cis-12 CLA isomers in CLA-fed geese compared to unsupplemented geese. However, it was noteworthy that CLA was deposited to a greater extent in liver lipids than in lipids in breast and leg muscles, reaching $2.82 \%, 1.88$ and $1.31 \%$ of total fatty acids, respectively, in geese fed $2.5 \%$ CLA.

Dietary CLA increased $(\mathrm{P}<0.01)$ the saturated fatty acid (SFA) concentration and decreased $(\mathrm{P}<0.05)$ the total monounsaturated fatty acids (MUFA) concentration in liver, leg and breast tissues. It also decreased $(\mathrm{P}<0.05)$ the total polyunsaturated fatty acids (PUFA) concentrations in leg and breast tissues. The palmitoleic, oleic, linoleic and linolenic acid concentrations tended to be lower $(\mathrm{P}<0.05)$ in CLA-fed animals than in animals not receiving CLA, while the concentrations of myristic, palmitic and stearic acids were higher $(\mathrm{P}<0.05)$. 
Table 6 The effects of dietary conjugated linoleic acid (CLA) treatments on the fatty acid composition of breast tissue in geese

\begin{tabular}{|c|c|c|c|c|}
\hline \multirow{3}{*}{ Fatty acid } & \multicolumn{4}{|c|}{ Fatty acids ( $\%$ of total) } \\
\hline & Group A & Group B & Group C & Group D \\
\hline & 0 & 0.5 & 1.5 & 2.5 \\
\hline C14:0 & $0.41^{\mathrm{b}} \pm 0.02$ & $0.49^{b} \pm 0.03$ & $0.62^{\mathrm{ab}} \pm 0.01$ & $1.06^{\mathrm{a}} \pm 0.08$ \\
\hline $\mathrm{C} 16: 0$ & $20.48^{b} \pm 2.59$ & $23.18^{b} \pm 2.82$ & $22.60^{b} \pm 2.49$ & $28.04^{\mathrm{a}} \pm 2.04$ \\
\hline $\mathrm{C} 16: 1, \mathrm{n}-7$ & $6.14 \pm 1.49$ & $5.76 \pm 1.25$ & $5.80 \pm 1.07$ & $5.01 \pm 1.20$ \\
\hline $\mathrm{C} 18: 0$ & $9.64^{\mathrm{c}} \pm 1.13$ & $12.55^{\mathrm{b}} \pm 1.09$ & $13.74^{\mathrm{a}} \pm 2.23$ & $13.25^{\mathrm{a}} \pm 1.19$ \\
\hline $\mathrm{C} 18: 1, \mathrm{n}-9$ & $37.11^{\mathrm{a}} \pm 3.90$ & $34.48^{b} \pm 2.58$ & $32.00^{b} \pm 2.91$ & $27.89^{c} \pm 3.07$ \\
\hline $\mathrm{C} 18: 2, \mathrm{n}-6$ & $20.12^{\mathrm{a}} \pm 3.02$ & $18.68^{b} \pm 3.19$ & $18.91^{\mathrm{b}} \pm 2.83$ & $16.87^{c} \pm 2.81$ \\
\hline $\mathrm{c}-9, \mathrm{t}-11 \mathrm{CLA}$ & ND & $0.28^{c} \pm 0.14$ & $0.59^{b} \pm 0.13$ & $1.02^{\mathrm{a}} \pm 0.18$ \\
\hline $\mathrm{t}-10, \mathrm{c}-12$ CLA & ND & $0.24^{\mathrm{c}} \pm 0.05$ & $0.48^{b} \pm 0.02$ & $0.86^{\mathrm{a}} \pm 0.07$ \\
\hline$\alpha \mathrm{C} 18: 3, \mathrm{n}-3$ & $0.73^{b} \pm 0.02$ & $0.66^{\mathrm{ab}} \pm 0.19$ & $0.71^{\mathrm{a}} \pm 0.22$ & $0.56^{\mathrm{b}} \pm 0.15$ \\
\hline $\mathrm{C} 20: 4, \mathrm{n}-6$ & $2.23^{\mathrm{a}} \pm 0.92$ & $1.42^{b} \pm 0.84$ & $1.54^{b} \pm 0.76$ & $0.89^{c} \pm 0.28$ \\
\hline$\Sigma$ CLA & ND & $0.52^{c} \pm 0.34$ & $1.07^{\mathrm{b}} \pm 0.56$ & $1.88^{\mathrm{a}} \pm 0.48$ \\
\hline$\Sigma$ SFA & $30.53^{c} \pm 3.45$ & $36.22^{b} \pm 3.19$ & $36.96^{\mathrm{b}} \pm 4.26$ & $42.35^{\mathrm{a}} \pm 4.28$ \\
\hline$\Sigma$ MUFA & $43.25^{\mathrm{a}} \pm 3.29$ & $40.24^{\mathrm{a}} \pm 3.02$ & $37.80^{b} \pm 2.19$ & $32.90^{b} \pm 3.02$ \\
\hline$\Sigma$ PUFA & $23.08^{\mathrm{a}} \pm 2.15$ & $21.28^{b} \pm 2.41$ & $22.23^{\mathrm{ab}} \pm 2.02$ & $20.20^{b} \pm 2.44$ \\
\hline UP & $3.14 \pm 0.13$ & $2.26 \pm 0.24$ & $3.01 \pm 0.36$ & $4.55 \pm 0.42$ \\
\hline
\end{tabular}

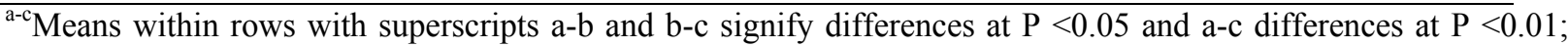
$\mathrm{n}=12$.

$\Sigma$ SFA - total saturated fatty acids; $\Sigma$ MUFA - total monounsaturated fatty acids; $\Sigma$ PUFA - total polyunsaturated fatty acids; $\Sigma$ CLA - total conjugated linoleic acids; UP - unidentified peaks; ND - not detectable.
}

Table 7 The effects of dietary conjugated linoleic acid (CLA) treatments on the fatty acid composition of leg tissue in geese

\begin{tabular}{|c|c|c|c|c|c|}
\hline \multirow{3}{*}{ Fatty acid } & \multirow[b]{3}{*}{ CLA $(\%)$} & \multicolumn{4}{|c|}{ Fatty acids ( $\%$ of total) } \\
\hline & & Group A & Group B & Group $\mathrm{C}$ & Group D \\
\hline & & 0 & 0.5 & 1.5 & 2.5 \\
\hline C14:0 & & $1.15^{b} \pm 0.18$ & $1.51^{\mathrm{b}} \pm 0.29$ & $1.96^{\mathrm{a}} \pm 0.21$ & $2.23^{\mathrm{a}} \pm 0.31$ \\
\hline C16:0 & & $19.23^{b} \pm 1.29$ & $19.19^{b} \pm 1.32$ & $20.40^{b} \pm 2.15$ & $24.62^{\mathrm{a}} \pm 1.38$ \\
\hline C16:1, n-7 & & $5.96 \pm 0.98$ & $5.61 \pm 0.63$ & $5.35 \pm 0.50$ & $5.02 \pm 0.62$ \\
\hline C18:0 & & $4.89^{c} \pm 0.39$ & $5.75^{c} \pm 0.53$ & $8.69^{b} \pm 0.39$ & $9.28^{\mathrm{a}} \pm 0.32$ \\
\hline C18:1, n-9 & & $43.42^{a} \pm 2.47$ & $43.57^{\mathrm{a}} \pm 2.87$ & $40.46^{\mathrm{ab}} \pm 3.05$ & $38.70^{\mathrm{b}} \pm 3.92$ \\
\hline $\mathrm{C} 18: 2, \mathrm{n}-6$ & & $19.20^{\mathrm{a}} \pm 2.38$ & $17.34^{\mathrm{ab}} \pm 2.19$ & $17.47^{\mathrm{ab}} \pm 2.57$ & $15.67^{b} \pm 2.75$ \\
\hline c-9, t-11 CLA & & ND & $0.09^{c} \pm 0.03$ & $0.32^{b} \pm 0.01$ & $0.71^{\mathrm{a}} \pm 0.08$ \\
\hline $\mathrm{t}-10, \mathrm{c}-12$ CLA & & ND & $0.13^{c} \pm 0.01$ & $0.27^{b} \pm 0.03$ & $0.60^{\mathrm{a}} \pm 0.07$ \\
\hline$\alpha \mathrm{C} 18: 3, \mathrm{n}-3$ & & $1.12^{\mathrm{a}} \pm 0.05$ & $0.94^{\mathrm{b}} \pm 0.04$ & $0.83^{b} \pm 0.02$ & $0.64^{c} \pm 0.06$ \\
\hline $\mathrm{C} 20: 4, \mathrm{n}-6$ & & $2.03^{b} \pm 0.49$ & $1.67^{\mathrm{ab}} \pm 0.23$ & $1.26^{\mathrm{b}} \pm 0.19$ & $0.95^{c} \pm 0.38$ \\
\hline$\Sigma$ CLA & & ND & $0.22^{\mathrm{c}} \pm 0.03$ & $0.59^{b} \pm 0.45$ & $1.31^{\mathrm{a}} \pm 0.39$ \\
\hline$\Sigma$ SFA & & $25.27^{c} \pm 3.49$ & $26.45^{c} \pm 2.35$ & $31.05^{b} \pm 3.28$ & $36.13^{a} \pm 3.78$ \\
\hline$\Sigma$ MUFA & & $49.38^{\mathrm{a}} \pm 4.29$ & $49.18^{a} \pm 3.97$ & $45.81^{b} \pm 4.02$ & $43.72^{b} \pm 4.18$ \\
\hline$\Sigma$ PUFA & & $22.35^{\mathrm{a}} \pm 1.42$ & $19.95^{\mathrm{ab}} \pm 1.65$ & $19.56^{\mathrm{ab}} \pm 1.89$ & $17.26^{b} \pm 1.94$ \\
\hline UP & & $3.00^{\mathrm{ab}} \pm 0.56$ & $4.20^{\mathrm{a}} \pm 0.78$ & $2.99^{\mathrm{ab}} \pm 0.83$ & $1.58^{b} \pm 0.39$ \\
\hline
\end{tabular}

\footnotetext{
${ }^{\mathrm{a}-\mathrm{c}}$ Means within rows with superscripts $\mathrm{a}-\mathrm{b}$ and $\mathrm{b}$-c signify differences at $\mathrm{P}<0.05$ and a-c differences at $\mathrm{P}<0.01$; $\mathrm{n}=12$.

$\Sigma$ SFA - total saturated fatty acids; $\Sigma$ MUFA - total monounsaturated fatty acids; $\Sigma$ PUFA - total polyunsaturated fatty acids; $\Sigma$ CLA - total conjugated linoleic acids; UP - unidentified peaks; ND - not detectable.
} 


\section{Discussions}

Conflicting results have been reported on the effect of CLA on growth and feed efficiency in different species (Thiel-Cooper et al., 2001; Wiegand et al., 2002; Dugan et al., 2004). In the present study increasing dietary CLA levels $(0 \%, 0.5 \%$ and $1.5 \%)$ did not affect $(\mathrm{P}>0.05)$ body weight and body weight gain of geese, but their feed intake, FCR and AFP decreased $(\mathrm{P}<0.05)$ compared with that of the control. However, CLA can inhibit growth at high dietary levels $(2.5 \%)$ of inclusion, which can be attributed to the sharp decrease in feed intake ( $\mathrm{P}<0.01)$. It has been shown by Cao et al. (2007) that the relationship between weight loss and CLA taken as a supplements or when occurring naturally in foods could be more complex than initially thought. They indicated that the anorectic effects of CLA are mainly exerted on the arcuate nucleus (ARC). The Agouti-related protein (AgRP) concentration and neusopeptide Y (NPY) mRNA expression were decreased upon CLA treatment, indicating that the metabolism of this specific lipid within the lateral hypothalamic neurons exerts a negative feedback on food intake.

Szymczyk et al. (2001) reported that in chicken there was a significant decrease (from $2.68 \%$ to $1.78 \%$ ) in AFP when CLA levels in the diet increased from $0 \%$ to $1.5 \%$. Sisk et al. (2001) reported that there was a dose-dependent effect of CLA in the regulation of body fat tissue in rats, so there would be an interference in the metabolism of fat and a reduction in body fat mass in rats if CLA was fed for an extensive period (Tsuboyama-Kasaoka et al., 2000). At the end of the present study, inclusion of CLA at different levels reduced $(\mathrm{P}<0.01)$ the AFP of geese from $1.57 \%$ to $0.5 \%$. It is evident that CLA is especially important in the catabolism of abdominal fat. Supplementation of CLA did not affect the body weight of geese. This could suggest that there is not a reduction of body weight but anorectic effects that led to the decreased AFP.

It has been shown that abnormally high serum levels of LDL-C and low serum levels of HDL-C are associated with an increased risk for atherosclerosis in rats (Yang et al., 2006). In the present study the serum lipid status of the CLA-fed geese showed decreased concentrations of serum TC, TG, and LDL-C, whereas the HDL-C concentration was increased compared with the control. The AI, defined as the ratio of TC/HDL-C and HDL-C, is believed to be an important risk factor of atherosclerosis. Our data clearly demonstrated that CLA supplementation leads to a decreased ratio compared with the unsupplemented control $(\mathrm{P}<0.05)$.

Mounting evidence has suggested that LPL is essentially an antiatherogenic enzyme (Kastelein et al., 2000; Fan et al., 2001). In comparison with the control, the LPL activity in the serum of geese fed CLA in our study was found to increase $(\mathrm{P}<0.05)$, which may play a key role in the lowering of AI. Our study suggests that the decrease in serum lipid concentration might result, at least to some extent, from an increased LPL activity. LPL is a glycoprotein involved in the transformation of dietary lipids into sources of energy for peripheral tissues. It separates free fatty acids from TGs present in chylomicrons and very low density lipoproteins. On the other hand, the hydrolysis of TG-rich lipoproteins also releases apolipoproteins and phospholipids that are precursors of high density lipoproteins (HDL). High density lipoproteins are key components of reverse cholesterol transport, bringing cholesterol from peripheral cells to the liver, where it is excreted in the bile (Yang et al., 2006). As a result, an efficient LPL is associated with lower TG and LDL levels but higher HDL levels, and is therefore potentially atheroprotective (Brunzell, 1995).

The changes in fatty acid profile in relation to CLA supplementation are consistent with those observed in studies of CLA supplementation in pigs (Joo et al., 2002; Ostrowska et al., 2003), pigeons (Aydin, 2005) and quails (Aydin \& Cook, 2004). The lowered MUFA levels and increased SFA levels in CLA-fed animals are probably due to an inhibition of $\triangle 9$-desaturase by CLA, since other in vivo and in vitro studies have shown that CLA reduces $\Delta 9$-desaturase activity either by suppressing mRNA (Ntambi \& Miyazaki, 2004) or by reducing the activity of the enzyme (Choi et al., 2000; Choi et al., 2001) thereby reducing MUFA levels. Corino et al. (2007) reported a lowered $\Delta 9$-desaturase in CLA-fed pigs.

\section{Conclusions}

We found that dietary supplementation of CLA to geese decreased feed intake, FCR and AFP, and also modified lipid content and fatty acid composition of fat in the body. The deposition of biologically active cis-9, trans-11 CLA and trans-10, cis-12 CLA isomers in CLA-fed geese suggests that the nutritional quality of geese products for human consumption is improved. 


\section{Acknowledgements}

The research was supported by the National "fifteen" science and technology key task (Serial Number: 2004BA514A09-3) and agricultural fine breed industrialization of Shandong province, China.

\section{References}

Aydin, R., 2005. The effect of conjugated linoleic acid on the fatty acid composition of different tissues and yolk lipids in pigeons. S. Afr. J. Anim. Sci. 35, 253-260.

Aydin, R. \& Cook, M.E., 2004. The effect of dietary conjugated linoleic acid on egg yolk fatty acids and hatchability in Japanese quail. Poult. Sci. 83, 2016-2022.

Badinga, L., Selberg, K.T., Dinges, A.C., Comer, C.W. \& Miles, R.D., 2003. Dietary conjugated linoleic acid alters hepatic lipid content and fatty acid composition in broiler chickens. Poult. Sci. 82, 111-116.

Brown, J.M., Halvorsen, Y.D., Lea-Currie, Y.R., Geigerman, C. \& McIntosh, M., 2001. Trans-10, cis-12, but not cis-9, trans-11, conjugated linoleic acid attenuates lipogenesis in primary cultures of stromal vascular cells from human adipose tissue. J. Nutr. 131, 2316-2321.

Brunzell, J.B., 1995. Familial lipoprotein lipase deficiency and other causes of the chylomicronemia syndrome. In: The Metabolic Basis of Inherited Diseases. Eds. Serivier, C.R., Beaudet, A.L., Sly, W.S. \& Valle, D., Volume II (7th ed). New York. pp. 1913-1932.

Cao, Z.P., Wang, F., Xiang, X.S., Cao, R., Zhang, W.B. \& Gao, S.B., 2007. Intracerebroventricular administration of conjugated linoleic acid (CLA) inhibits food intake by decreasing gene expression of NPY and AgRP. Neurosci. Lett. 418, 217-221.

Chin, S.D., Liu, W., Storkson, J.M., Ha, Y.L. \& Pariza, M.W., 1992. Dietary sources of conjugated dienoic isomers of linoleic acid, a newly recognized class of anticarcinogens. J. Food Compos. Anal. 5, 185-197.

Choi, Y., Kim, Y.C., Han, Y.B., Park, Y., Pariza, M.W. \& Ntambi, J.M., 2000. The trans-10, cis-12 isomer of conjugated linoleic acid downregulates stearoyl-CoA desaturase I gene expression in 3T3-L1 adipocytes. J. Nutr. 130, 1920-1924.

Choi, Y., Park, Y., Pariza, M.W. \& Ntambi, J.M., 2001. Regulation of stearoyl-CoA desaturase activity by the trans-10, cis-12 isomer of conjugated linoleic acid in HepG2 cells. Biochem. Bioph. Res. Co. 284, 689-693.

Christie,W.W., 1982. Lipid Analysis (Version 2). Pergamon Press,Oxford. 207 p.

Corino, C., Lo Fiego, D.P., Macchioni, P., Pastorelli, G., Di Giancamillo, A., Domeneghini, C. \& Rossi, R., 2007. Influence of dietary conjugated linoleic acids and vitamin $\mathrm{E}$ on meat quality, and adipose tissue in rabbits. Meat Sci. 76, 19-28.

Delany, J.P., Blohm, F., Truett, A.A., Scimeca, J.A. \& West, D.B., 1999. Conjugated linoleic acid rapidly reduces body fat content in mice without affecting energy intake. Am. J. Phys. 276, R1172-1179.

Dugan, M.E.R., Aalhus, J.L. \& Kramer, J.K.G., 2004. Conjugated linoleic acid pork research. Am. J. Clin. Nutr. 79, 1212-1216.

Evans, M., Brown, J. \& McIntosh, M., 2002. Isomer-specific effects of conjugated linoleic acid (CLA) on adiposity and lipid metabolism. J. Nutr. Biochem. 13, 508-516.

Fan, J., Unoki, H., Kojima, N., Sun, H., Shimoyamada, H. \& Deng, H., 2001. Overexpression of lipoprotein lipase in transgenic rabbits inhibits diet induced hypercholesterolemia and atherosclerosis. J. Biol. Chem. 276, 40071-40079.

Folch, J., Lees, M. \& Sloane-Stanley, G.H., 1957. A simple method for the isolation and purification of total lipides from animal tissues. J. Biol. Chem. 226, 497-509.

Joo, S.T., Lee, J.I., Ha, Y.L. \& Park, G.B., 2002. Effects of dietary conjugated linoleic acid on fatty acid composition, lipid oxidation, color, and water-holding capacity of pork loin. J. Anim. Sci. 801, 108-112.

Kastelein, J.J., Jukema, J.W., Zwinderman, A.H., Clee, S., Van Boven, A.J. \& Jansen, H., 2000. Lipoprotein lipase activity is associated with severity of angina pectoris. Circulation 102, 1629-1633.

Kennedy, S.R., Campbell, P.J, Porter, A. \& Tocher, D.R., 2005. Influence of dietary conjugated linoleic acid (CLA) on lipid and fatty acid composition in liver and muscle of Atlantic salmon (Salmo salar). Comp. Biochem. Phys. B. 141, 168-178. 
Kritchevsky, D., Tepper, S.A., Wright, S., Tso, P. \& Czarnecki, S.K., 2000. Influence of graded levels of conjugated linoleic acid (CLA) on experimental atherosclerosis in rabbits. Nutr. Res. 22 (11), 1275-1279.

Lee, K.N., Kritchevsky, D. \& Pariza, M.W., 1994. Conjugated linoleic acid and atherosclerosis in rabbits. Atherosclerosis 108, 19-25.

Li, Y. \& Watkins, B.A., 1998. Conjugated linoleic acids alter bone fatty acid composition and reduce ex vivo prostaglandin E2 biosynthesis in rats fed n-6 or n-3 fatty acids. Lipids 33, 417-425.

Nicolosi, R.J., Rogers, E.J., Kritchevski, D., Scimeca, J.A. \& Huth, P.J., 1997. Dietary conjugated linoleic acid reduces plasma lipoproteins and early aortic atherogenesis in hypercholesterolemic hamsters. Artery 22, 266-277.

Ntambi, J.M. \& Miyazaki, M., 2004. Regulation of stearoyl-CoA desaturases and role in metabolism. Prog. Lipid Res. 43, 91-104.

Ohnuki, K., Haramizu, S., Oki, K., Ishihara, K. \& Fushiki, T., 2001. A single oral administration of conjugated linoleic acid enhanced energy metabolism in mice. Lipids 36, 583-587.

Oku, H., Wongtangtintharn, S., Iwasaki, H. \& Toda, T., 2003. Conjugated linoleic acid (CLA) inhibits fatty acid synthetase activity in vitro. Biosci. Biotech. Bioch. 67, 1584-1586.

Ostrowska, E., Cross, R.F., Muralitharan, M., Bauman, D.E. \& Dunshea, F.R., 2003. Dietary conjugated linoleic acid differentially alters fatty acid composition and increases conjugated linoleic acid content in porcine adipose tissue. Br. J. Nutr. 90, 915-928.

Pariza, M.W., Park, Y. \& Cook, M.E., 2001. The biological active isomers of conjugated linoleic acid. Prog. Lipid Res. 40, 283-298.

Park, Y., Albright, K.J., Liu, W., Storkson, J.M., Cook, M.E. \& Pariza, M.W., 1997. Effect of conjugated linoleic acid on body composition on mice. Lipids 32, 853-858.

SAS, 2001. Statistical Analysis Systems User's Guide (8.2th ed.). SAS Institute Inc., Cary, North Carolina.

Sisk, M., Hausman, D., Martin, R. \& Azain, M., 2001. Dietary conjugated linoleic acid reduces adiposity in lean but not obese Zucker rats. J. Nutr. 131, 1668-1674.

Szymczyk, B., Pisulewski, P., Szczurek, M. \& Hanczakowski, P., 2001. Effects of conjugated linoleic acid on growth performance, feed conversion efficiency, and subsequent carcass quality in broiler chickens. J. Nutr. 85, 465-473.

Takahashi, Y., Kushiro, M., Shinohara, K. \& Ide, T., 2003. Activity and mRNA levels of enzymes involved in hepatic fatty acid synthesis and oxidation in mice fed conjugated linoleic acid. Biochim. Bioph. Acta. 1631, 265-273.

Terpstra, A.H., Beynen, A.C., Everts, H., Kocsis, S., Katan, M.B. \& Zock, P.L., 2002. The decrease in body fat in mice fed conjugated linoleic acid is due to increases in energy expenditure and energy loss in the excreta. J. Nutr. 132, 940-945.

Thiel-Cooper, R.L., Parrish Jr., F.C., Sparks, J.C., Wiegand, B.R. \& Ewan, R.C., 2001. Conjugated linoleic acid changes swine performance and carcass composition. J. Anim. Sci. 79, 1821-1828.

Tsuboyama-Kasaoka, N., Takahashi, M., Tanemura, K., Kim, H.J., Tange, T., Okuyama, H., Kasai, M., Ikemoto, S. \& Ezaki, O., 2000. Conjugated linoleic acid supplementation reduces adipose tissue by apoptosis and develops lipodystrophy in mice. Diabetes 49, 1534-1542.

Wang, Y. \& Jones, P.J., 2004. Dietary conjugated linoleic acid and body composition. Am. J. Clin. Nutr. 79, $1153-1158$.

Wiegand, B.R., Sparks, J.C., Parrish Jr., F.C. \& Zimmerman, D.R., 2002. Duration of feeding conjugated linoleic acid influences growth performances, carcass traits and meat quality of finishing barrows. J. Anim. Sci. 80, 637-643.

Wilson, D.E. \& Spiger, M.J., 1973. A dual precipitation method for quantitative plasma lipoprotein measurement without ultracentrifugation. J. Lab. Clin. Med. 82, 473-482.

Yamasaki, M., Ikeda, A., Oji, M., Tanaka, Y., Hirao, A., Kasai, M., Iwata, T., Tachibana, H. \& Yamada, K., 2003. Modulation of body fat, and serum leptin levels by dietary conjugated linoleic acid in SpragueDawley rats fed various fat-level diets. Nutr. 19, 30-35.

Yang, R.L., Le, G.W., Li, A.L., Zheng, J.L. \& Shi, Y.H., 2006. Effect of antioxidant capacity on blood lipid metabolism and lipoprotein lipase activity of rats fed a high-fat diet. Nutr. 22, 1185-1191. 